\title{
Muskelkrank und lebensstark
}

\section{Erhard Taverna}

erhard.taverna@saez.ch

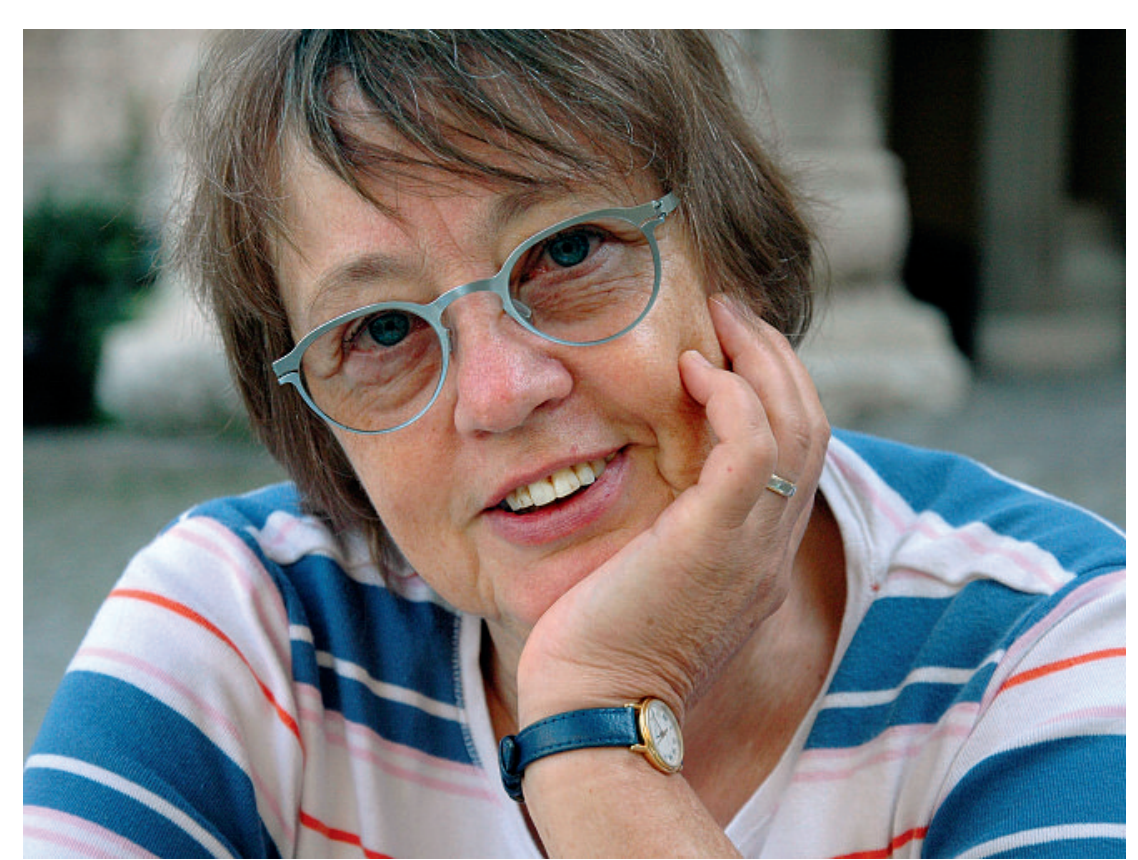

Erica Brühlmann-Jecklin hat die Schweizerische Gesellschaft für Muskelkranke SGMK initiiert und jahrzehntelang als Geschäftsführerin zum Erfolg geführt.

Selbstbestimmt und gleichgestellt sollen Menschen mit Muskelkrankheiten leben können. In den 1970er Jahren begannen erstmals Kranke und Behinderte, ihre Interessen unabhängig und eigenständig vorzutragen. Sie organisierten Selbsthilfegruppen, Beratungsstellen und Vereine oder schrieben als Betroffene über das eigene Sterben oder ihre Behinderungen. Diese zahlreichen Eigeninitiativen veränderten entscheidend die öffentliche und politische Wahrnehmung. Der neue Umgang mit bisher vernachlässigten Mitmenschen hat auch Medizin und Pflege verändert. Ein Mitspracherecht ist heute so selbstverständlich wie die Lobbyarbeit zugunsten neuer Gesetze und baulicher Vorschriften, für technische Hilfsmittel oder die Finanzierung von Forschungsvorhaben, für medizinische Einrichtungen oder für Pilotprojekte wie die Assistenzpflege.

\section{Die Gründerin}

Erica Brühlmann-Jecklin, geboren 1949, hatte unfreiwillig Gelegenheit, im alten und neuen Balgrist über ihre Situation als Muskelerkrankte nachzudenken. Erstens spürte sie, dass die damalige Diagnose einer «progressiven Muskeldystrophie» nicht stimmen konnte, zweitens wollte sie trotz allem den Beruf der Krankenschwester erlernen und drittens Menschen in der gleichen Situation zusammenbringen. Als junge Lehrerin für Pflegende schrieb sie 1973 an alle Chefärzte neuro- logischer Kliniken einen Brief, in dem sie um Mithilfe bei der Gründung einer «Gesellschaft für Dystrophiker» bat. Der Rücklauf war phänomenal und durchwegs ermunternd, doch niemand hatte Zeit, ausser dem damaligen Leiter der neurologischen Poliklinik Dr. med. Felix Jerusalem (1932-1996). Er war bald darauf Präsident des ersten Vorstandes der am 15. Juni 1974 gegründeten Schweizerischen Gesellschaft für Muskelkrankheiten (später: Muskelkranke) SGMK [1]. Aus einer Regionalstelle für die französische Schweiz entstand 1977 die Association Suisse Romande contre la Myopathie ASRM. Ursprünglich wollte Frau Brühlmann-Jecklin nur Gleichgesinnte vereinigen, doch dann stand sie als erste Geschäftsführerin der Gesellschaft vor einem riesigen Problemberg.

Da waren überarbeitete Eltern erkrankter Kinder ohne Aussicht auf Entlastung, es fehlte an sachgerechtem Wissen und an Kontakten, es gab weder Ferien für Betroffene noch Ausbildungsmöglichkeiten für Schulabgänger. Berufs-, Wohn- und Hilfsmittelfragen waren ungeklärt, Fehlplazierungen in ungeeigneten Heimen oft die Regel. Mit der korrigierten Diagnose einer «Congenitalen Myopathie» war Erica Brühlmann in der Lage, in jahrelanger Aufbauarbeit mit Hilfe ihres Ehemannes und zahlreicher Freiwilliger - ein enormes Pensum zu bewältigen. Der Mitstreiterin und Freundin Ursula Eggli bleibt sie besonders verbunden. Die 2008 Verstorbene war Mitglied und zeitweise im Vorstand. Sie wehrte sich gegen die ausgrenzende «Ideologie der Normalität» mit ihrem 1977 veröffentlichten Buch «Herz im Korsett». Ein Tagebuch, das weit über die Grenzen hinaus beachtet wurde.

Erica Brühlmann arbeitet heute als Psychotherapeutin in einer Praxisgemeinschaft in Schlieren. Beim Erzählen im Innenhof des Landesmuseums kommt sie richtig in Fahrt. Sie ist voller Geschichten und Anekdoten. Als begabte Organisatorin und Vernetzerin kennt sie alle und jeden, der für ihre Anliegen in Politik und Wissenschaft etwas zu sagen hat. Sie und ihre Nachfolger haben unzählige Familien beraten, Ferienlager betreut, Fortbildungen für Patienten und Ärzte organisiert, Kontakt- und Selbsthilfegruppen unterstützt, Aufklärungskampagnen gestartet und Sponsoren angeworben. 2002 initiierte die Gesellschaft das Projekt «Regionale Muskelzentren Schweiz». 2006 wurde am Kantonsspital St. Gallen das erste einer ganzen Reihe geplanter Zentren eröffnet. Parallel dazu soll das Netzwerk «Myosuisse» Fachpersonen und Spezialisten zusammenführen. Als politische Aktivistin hat sie 1981 an der ersten grossen Demonstration von Behinderten in Bern Lieder gesungen, die sie selber kompo- 
niert und getextet hat. Sie singt und spielt Gitarre, Klavier, Geige, Trompete, Mundharmonika und Perkussion. Ein Ein-Frau-Konzert mit Radioauftritten und CD-Ausgaben.

Ganz nebenbei schreibt die Traumaspezialistin auch Bücher. Ein Buch über Anatomie und Physiologie für Pflegeberufe wurde als Lehrmittel schon $13 \mathrm{Mal}$ aufgelegt. Ein kritisches Werk wie «Irren ist ärztlich» [2] wurde 1986 mit dem Luzerner Literaturpreis ausgezeichnet, ihr «Amalgam-Report» sorgte 1990 für landesweite Diskussionen. Sie hat auch Kinderbücher wie «Balz und Bettina» oder «Ümit will ein Doktor werden» geschrieben. Heute begeht ihr Kind, wie sie den Verein einmal nennt, den 35. Geburtstag. Sie ist zufrieden, der Verein habe alle Turbulenzen gut überstanden, habe sich ständig professionalisiert und leiste eine hervorragende Arbeit. Zeit für weitere Bücher, wie das vorläufig letzte aus dem Jahr 2009, eine Familiengeschichte über drei Generationen aus dem Prättigau, das die in Küblis geborene Autorin bestens kennt.

Noch eine kritische Anmerkung, die sich Frau Brühlmann in ihrer Bescheidenheit niemals erlauben würde: Zahlreiche ehemalige ärztliche Mitarbeiter verdanken den Erfahrungen, die ihnen auch die SGMK ermöglichte, wissenschaftliche Arbeiten, akademische Karrieren, Preise und Ehrungen. Die Gründerin wurde zu keiner Zeit öffentlich geehrt. Sie nimmt es gelassen, mit Humor. Ein Grund mehr für eine längst verdiente Aufmerksamkeit. Vielleicht einmal von ärztlicher Seite?

\section{Das Buch}

Im Auftrag der Schweizerischen Gesellschaft für Muskelkranke SGMK ist zum 35. Jubiläumsjahr ein Buch entstanden. Es konnte dank Sicherung der Finanzierung mit Geldern des Lotteriefonds verschiedener Kan-

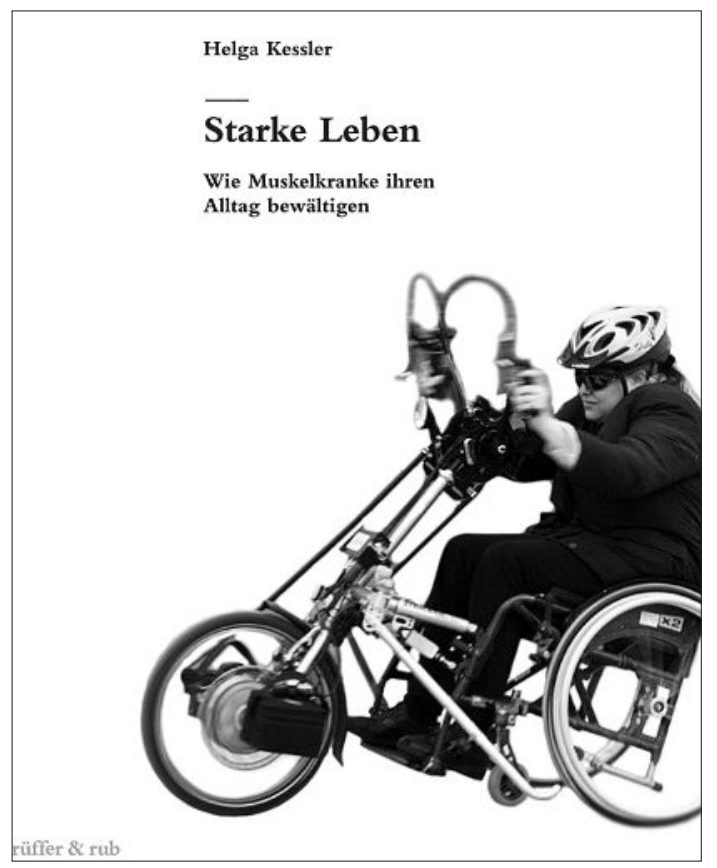

tone, swisslos, Kulturproduzent Migros und weiterer Stiftungen lanciert werden. «Starke Leben - Wie Muskelkranke ihren Alltag bewältigen» [3] ist der Titel eines inhaltlich und gestalterisch hervorragend gelungenen Werkes. Gemäss Auftraggeber soll es einer breiten Öffentlichkeit näherbringen, was es bedeutet, muskelkrank zu leben. Die Autorin Helga Kessler, geboren 1956, studierte Biologin und Chemikerin, legt eine spannende und lehrreiche Recherche vor. Sie berichtet von elf Begegnungen mit unterschiedlich behinderten Muskelkranken. Einmalige Schilderungen, die in den längeren Ablauf einer Krankenbiografie eingebettet sind. Das Schicksal eines an ALS erkrankten 50-jährigen Mannes zieht sich wie ein roter Faden durch den ganzen Erzählstrang. Die Sprache ist klar, mitfühlend und respektvoll, nie larmoyant, ausschweifend oder beschönigend. Betroffene und Angehörige kommen ausgewogen zu Wort. Wünsche und Hoffnungen, Gefühle wie Ängste und Frustrationen, die Vereinsamung, der nahende Tod, die palliativen Möglichkeiten und der Ausweg über die Sterbehilfe sind offen angesprochen. Die Leidenden berichten von Wut und Trauer und lassen dabei auch unglaublich viel Lebensmut, Gelassenheit und Humor erkennen. Wie immer bei solchen Begegnungen sind es letztlich die Gesunden, die von den Kranken beschenkt und belehrt werden.

Die Momentaufnahmen, wie die Autorin ihre Porträts nennt, sind geschickt in elf Kapiteln angeordnet. Sie umfassen die Aspekte des Alltags wie Krankheit, Hobby, Arbeit, Familie, Kinder, Reisen, Wohnen, Mobilität oder Abschied. Den persönlichen Ausprägungen der Krankheit folgen die verallgemeinernden Darstellungen neuromuskulärer Erkrankungen. Auch für Mediziner eine lesenswerte Zusammenfassung aktuellen Wissens über Ätiologie, Prognose und Therapiemöglichkeiten der Friedreich-Ataxie, Myasthenia gravis, spinaler Muskelatrophien, Amyotrophen Lateralsklerosen, Polyneuropathien, neuralen Muskelatrophien, entzündlichen Muskelkrankheiten und der Muskeldystrophie/Duchenne.

Zum Buch, das auch bei der Gesellschaft für Muskelkranke bezogen werden kann, gehören eine CD [4] und Illustrationen aus einer Fotoreportage von Vera Markus. Sie hat die Autorin begleitet und zeigt ihre Aufnahmen an einer Foto-Wanderausstellung.

\section{Literatur}

1 Schweizerische Gesellschaft für Muskelkranke SGMK. Kanzleistrasse 80, 8004 Zürich. www.muskelkrank.ch

2 Brühlmann-Jecklin E. Irren ist ärztlich Analyse einer Krankengeschichte. Bern: Zytglogge; 1986. 183 Seiten.

3 Kessler H. Starke Leben - Wie Muskelkranke ihren Alltag bewältigen. Zürich: rüffer \& rub; 2009. 242 Seiten

4 CD von Erica Brühlmann-Jecklin, un pocchettino es birebitzeli. Bern: Zytglogge. www.ebj.ch 